\title{
Epidemiologia Comparativa da Pinta-Preta do Tomateiro sob Quatro Regimes de Pulverização*
}

\author{
Pierce A. Paul ${ }^{1}$, Francisco X. R. Vale ${ }^{1}$, Laércio Zambolim ${ }^{1}$, Paulo Cézar R. Fontes ${ }^{2}$, Reginaldo R. Coelho ${ }^{1} \&$ \\ Antonio J. Macabeu ${ }^{1}$ \\ ${ }^{1}$ Departamento de Fitopatologia e ${ }^{2}$ Fitotecnia, Universidade Federal de Viçosa, Viçosa, MG, CEP 36571-000, \\ e-mail: dovale@ufv.br
}

(Aceito para publicação em 25/11/2003)

Autor para correspondência: Pierce Anderson Paul

PAUL P. A., VALE, F.X.R., ZAMBOLIM, L., FONTES, P.C.R., COELHO, R.R. \& MACABEU, A.J. Epidemiologia comparativa da pintapreta do tomateiro sob quatro regimes de pulverização. Fitopatologia Brasileira 29:475-479. 2004.

\section{RESUMO}

Objetivando estudar o desenvolvimento da pinta-preta (Alternaria solani) do tomateiro (Lycopersicon esculentum) sob diferentes regimes de pulverização com calda Viçosa, um ensaio foi conduzido, utilizando os seguintes tratamentos: 1) aplicação semanal; 2) aplicação com base nos sistemas de previsão TOMCAST (VSD20); 3) CUFAST; e 4) FAST. As aplicações semanais foram iniciadas na segunda semana após o transplantio enquanto as outras foram feitas com base nos respectivos modelos de previsão. As avaliações da severidade foram feitas semanalmente com o auxílio de uma escala diagramática e os dados foram submetidos ao ajustamento dos modelos de Gompertz, logístico e monomolecular, por meio de análise de regressão linear. O modelo logístico se ajustou melhor aos dados e foi utilizado para determinar as taxas de progresso da doença $(r)$ que, juntamente com a AACPD, foram utilizadas para comparar os tratamentos. $\mathrm{O}$ tratamento semanal proporcionou menor taxa de progresso de doença e AACPD, sem, no entanto, diferir significativamente dos efeitos dos tratamentos baseados nos modelos TOMCAST e FAST. Comparados com o regime de pulverização semanal, os regimes baseados nestes dois modelos permitiram uma redução da severidade da pinta-preta com 40 a 60\% de redução no número de aplicações de fungicida.

Palavras-chave adicionais: Lycopersicon esculentum, sistema de previsão, modelo matemático.

\section{ABSTRACT}

Comparative epidemiology of tomato early blight on tomatoes under four different spraying regimes

A field trial was conducted in order to stud the development of tomato (Lycopersicon esculentum) early blight caused by Alternaria solani using four different spraying treatments: 1) weekly sprays; 2) application according to the TOMCAST (VSD20); 3) CUFAST; and 4) FAST forecasting system. The fungicide used was Viçosa Mixture, a nutrient amended Bordeaux mixture. Weekly sprays were begun in the second week after planting while the other spray treatmets were made according to each respective forecasting model. Disease severity was assessed weekly with the aid of a diagrammatic scale. Linear

\section{INTRODUÇÃO}

A importância da pinta-preta, causada por Alternaria solani Sorauer, cresce durante os meses mais quentes e úmidos, passando a ser um dos maiores problemas da cultura do tomateiro (Lycopersicon esculentum Mill.). As perdas na produção do tomateiro que ocorrem em virtude da doença podem ser tanto diretas quanto indiretas. Diretas pela infecção dos frutos e indiretas pela destruição da folhagem, levando à

*Parte da Tese de Mestrado do primeiro autor apresentada à Universidade Federal de Viçosa. (1999) regression analysis was used to evaluate accuracy-of-fit for the Gompertz, logistic, and monomolecular mathematical models and the disease progress data. The logistic model best described disease progress over time. The apparent infection rate (r), for the logistic model, and AUDPC values, were used to compare treatments. Disease progress rate (r) and AUDPC values for the weekly spraying treatment were the lowest. However, they were not statistically different from the treatments based on the TOMCAST and FAST schedules. Compared with the weekly spray regime to limit early blight severity, the spraying schedules associated with TOMCAST and FAST resulted in 40 to $60 \%$ fewer applications. escaldadura dos frutos expostos aos raios do sol.

$\mathrm{Na}$ ausência de variedades comerciais resistentes à pinta-preta, as medidas de controle têm-se baseado em aplicações sistemáticas de fungicidas protetores seguindo um calendário de aplicações semanais que não considera as condições favoráveis ao desenvolvimento das doenças. Isso tem causado o uso excessivo de fungicidas na cultura (Vale et al., 1992), o que pode aumentar o custo da produção e os resíduos nos frutos a serem comercializados, além de aumentar os danos ao meio ambiente e aos aplicadores.

Visando racionalizar a utilização de fungicidas para o 


\section{P.A. Paul et al.}

controle da pinta-preta do tomateiro, foram desenvolvidos sistemas de previsão com base nos dados meteorológicos (Madden et al., 1978; Pitblado, 1992; Sandlan \& Zitter, 1993) que visam identificar períodos favoráveis ao desenvolvimento da doença e com base nisso, determinar o início e os intervalos de aplicação de fungicidas.

Objetivou-se neste trabalho comparar quatro regimes de pulverização quanto aos seus efeitos sobre o desenvolvimento e eficiência no controle da pinta-preta do tomateiro.

\section{MATERIALEMÉTODOS}

O ensaio foi conduzido na área experimental da Universidade Federal de Viçosa de 16 de janeiro a 16 de abril de 1996. Foi utilizada a cultivar de tomate Santa Clara conduzida pelo sistema tradicional com espaçamento de 0,60 m entre plantas na fileira. Cada unidade experimental foi constituída de duas fileiras de 4,5 m, espaçadas de um metro entre si. O delineamento experimental utilizado foi o de blocos casualizados, com quatro tratamentos e três repetições. Os tratamentos foram constituídos do uso da Calda Viçosa em quatro regimes de pulverização. Um tratamento sem a aplicação de fungicida foi omitido a fim de se minimizar o erro critico ou representacional (Van der Plank, 1963; James et al., 1973; Zadoks \& Schein, 1979) devido à interferência entre parcelas.

A aplicação semanal teve início na segunda semana após o transplantio. As outras aplicações tiveram seus inícios e intervalos determinados pelos sistemas de previsão com base nas variáveis climáticas (Tabelas 1 e 2). No sistema TOMCAST (Tomato Forecasting) (Pitblado, 1992) valores de severidade de doença (VSD), que variam de 0 a 4 , foram calculados em função do molhamento foliar e da temperatura média do ar durante os períodos de molhamento (Tabela 1) proposta por Madden et al. (1978). Após o transplantio, para cada dia foi calculado o valor de VSD, e os valores diários foram acumulados até atingir o valor 20, quando então foi feita a primeira aplicação de fungicida. Iniciou-se nova acumulação de VSD's até atingir novamente 20 quando então foi feita a nova pulverização e assim sucessivamente.

O sistema CUFAST (Cornell University - FAST) (Sandlan \& Zitter, 1993) foi desenvolvido a partir do modelo FAST, modificado na Universidade de Cornell, alterando certos limites para tomada de decisão de se pulverizar sem a neces-

TABELA 1 - Valores de severidade de doença (VSD), como função da duração do período de molhamento foliar e da temperatura média do ar durante este período

\begin{tabular}{lllcccc}
\hline \hline \multirow{2}{*}{$\begin{array}{l}\text { Temperatura } \\
\text { média }\left({ }^{\circ} \mathbf{C}\right)\end{array}$} & \multicolumn{6}{c}{ Valores de Severidade da Doença(VSD) } \\
\cline { 2 - 7 } & $\mathbf{0}$ & $\mathbf{1}$ & $\mathbf{2}$ & $\mathbf{3}$ & $\mathbf{4}$ \\
\hline 13,0 & 17,5 & $0-6 \mathrm{~h}^{*}$ & $7-15$ & $16-20$ & $21+$ & \\
17,6 & 20,5 & $0-3$ & $4-8$ & $9-15$ & $16-22$ & $23+$ \\
20,6 & 25,5 & $0-2$ & $3-5$ & $6-12$ & $13-20$ & $21+$ \\
25,6 & 29,5 & $0-3$ & $4-8$ & $9-15$ & $16-22$ & $23+$ \\
\hline
\end{tabular}

Fonte: Madden et al. (1978)

*Duração do período de molhamento foliar sidade de se determinar o período de molhamento foliar. Neste modelo, o molhamento foliar foi calculado por um método desenvolvido por J.R. Pelletier. Este método utiliza a chuva, horas de umidade relativa maior que $90 \%$ e umidade relativa mínima em dois dias consecutivos. O programa CUFAST foi implementado para ser utilizado em computador IBM-PC. Os dados climáticos foram armazenados em computador e, utilizando-se o programa CUFAST, foram determinados o início e os intervalos das aplicações de fungicidas.

O sistema de previsão FAST (Forecasting Alternaria solani on Tomato) (Madden et al., 1978) incorpora dois modelos diferentes de se calcular a severidade potencial da pinta-preta: no primeiro (orvalho/temperatura), as horas de molhamento foliar e temperatura média do ar durante o molhamento foram combinadas para se obterem os valores de severidade (S) (Tabela 1) e, no segundo modelo (chuva/ temperatura/umidade relativa), valores diários de taxas de severidade (R) foram determinados a partir de medições de três variáveis ambientais (Tabela 2). Os valores de R foram determinados com base nos valores médios de temperatura do ar dos últimos cinco dias; horas de umidade relativa maior que 90\% dos últimos cinco dias; e total de chuva dos últimos sete dias. A primeira aplicação foi recomendada quando o total dos valores de S (TS), registrados desde o início da estação de cultivo, alcançou o nível crítico de 35 e as plantas estavam no campo por pelo menos 35 dias. Subseqüentemente, as aplicações de fungicidas foram feitas quando os valores cumulativos de $S$ (CS) $\geq 14$ : aplicação em intervalos de sete dias, e, quando $C S \geq 14$ e os valores cumulativos de $R(C R) \geq 9$ : aplicação em intervalos de cinco dias.

Foi instalado, entre as plantas, um abrigo dentro do qual foi colocado um termo-higro-humectógrafo (G. Lufft GmbH \& Co) para registrar os dados de molhamento foliar, umidade relativa e temperatura. Os dados de chuva foram coletados diariamente de um pluviômetro instalado a cerca de 200 m do local do ensaio.

Foram feitas avaliações semanais da percentagem de

TABELA 2 - Taxas de severidade de doença (R) em função da temperatura média do ar, da umidade relativa (UR) maior que $90 \%$ e total de chuva

\begin{tabular}{|c|c|c|c|}
\hline $\begin{array}{l}\text { Temperatura } \\
\text { média }\left({ }^{\circ} \mathbf{C}\right)^{\mathrm{a}}\end{array}$ & $\begin{array}{c}\text { Horas } \\
\text { UR }>90 \% \\
\end{array}$ & $\begin{array}{c}\text { Total } \\
\text { Chuva }\end{array}$ & $\mathbf{R}^{\mathrm{d}}$ \\
\hline$<22$ & $<60$ & $<2.5$ & 0 \\
\hline$>22$ & $<60$ & $<2.5$ & 0 \\
\hline$<22$ & $>60$ & $<2.5$ & 1 \\
\hline$<22$ & $<60$ & $>2.5$ & 1 \\
\hline$<22$ & $>60$ & $>2.5$ & 1 \\
\hline$>22$ & $>60$ & $<2.5$ & 2 \\
\hline$>22$ & $<60$ & $>2.5$ & 2 \\
\hline$>22$ & $>60$ & $>2.5$ & 3 \\
\hline $\begin{array}{l}\text { Fonte: Madden et a } \\
\text { - Temperatura méd } \\
\text { - Horas de umidade } \\
\text { - Total de chuva no } \\
\text { - Valores da taxa de }\end{array}$ & $\begin{array}{l}78 \text { ) } \\
\text { ra os últimos cin } \\
\text { tiva }(>90 \%) \text { no } \\
\text { mos sete dias (c } \\
\text { eridade de doens }\end{array}$ & $\begin{array}{l}\left.{ }^{\circ} \mathrm{C}\right) \text {; } \\
\text { cinco dias; }\end{array}$ & \\
\hline
\end{tabular}


Epidemiologia comparativa da pinta-preta do tomateiro sob quatro...

área foliar lesionada, atribuindo notas de severidade com base em uma escala diagramática (Boff, 1988). Os dados de severidade foram utilizados para determinar os valores de Área Abaixo da Curva de Progresso da Doença (AACPD) para cada tratamento e estes foram, então, submetidos à análise de variância utilizando o programa SAS.

Para comparar os tratamentos, junto com a AACPD, foram determinadas as taxas de progresso da doença (r). Para tal, os dados de severidade foram submetidos ao ajustamento de três modelos matemáticos por meio de análise de regressão linear utilizando o programa SAS. O modelo que melhor se ajustasse ao progresso da doença foi selecionado pelo coeficiente de determinação $\left(\mathrm{R}^{* 2}\right)$ e pelo quadrado médio do resíduo (QMR*). Os modelos testados foram: monomolecular, $\mathrm{y}=1-\left(1-\mathrm{y}_{0}\right) e^{-\mathrm{rt}}$; logístico, $\mathrm{y}=1 /\left(1+\left[\left(1-\mathrm{y}_{0}\right) / \mathrm{y}_{0}\right] \mathrm{e}^{(-\mathrm{rt})}\right)$ e gompertz, $\mathrm{y}=e^{(-\ln (\mathrm{y}) \exp (-\mathrm{rt}))}$, em que $\mathrm{y}=$ quantidade de doença, $\mathrm{y}_{0}=$ quantidade inicial de doença, $r=$ taxa de progresso da doença e $\mathrm{t}$ = tempo em dias após o transplantio.

Para relacionar as variáveis climáticas com o progresso da doença, foram estimadas as taxas de infecção aparente no intervalo de sete dias $\left(\mathrm{r}_{7}\right)$ por meio da unidade logit, $\mathrm{r}_{7}=(\operatorname{logit}$ $\mathrm{y}_{2}$-logit $\left.\mathrm{y}_{1}\right) /\left(\mathrm{t}_{2}-\mathrm{t}_{1}\right)$ e estas foram plotadas paralelamente ao curso das variáveis microclimáticas.

\section{RESULTADOSE DISCUSSÃO}

O período de condução deste ensaio foi caracterizado por condições de altas temperaturas e umidade. Houve uma predominância de temperaturas maiores que $25^{\circ} \mathrm{C}$ com duração média semanal em torno de 8 h (Figura 1D). A duração média semanal de molhamento foliar e umidade relativa maior que $90 \%$ foi de 10,67 e 10,88 h, respectivamente (Figura 1C). Nas seis semanas finais do ciclo de cultura, as horas de umidade relativa maior que $90 \%$ e molhamento foliar aumentaram, com as respectivas médias semanais sendo de 12,45 e 12,70 h. As chuvas foram freqüentes, de baixa intensidade e relativamente bem distribuídas ao longo do período deste ensaio (Figura 1B).

Relatos feitos na literatura sobre o efeito das condições climáticas no desenvolvimento da pinta-preta do tomateiro sugerem que epidemias severas ocorrem com maior freqüência sob condições de temperaturas maiores que $25^{\circ} \mathrm{C}$, associadas a alta umidade (Kranz, 1977; Maffia et al., 1980; Jones et al., 1991; Rotem, 1994). As semanas de chuvas de maior intensidade e a época de aumento da duração das horas de umidade relativa maior que $90 \%$ e molhamento foliar coincidiram com a fase durante a qual a taxas de infecção aparente foram maiores (Figura 1A). Nunes et al. (1984) observaram maiores taxas de infecção aparente de $A$. solani, na cultura da batateira (Solanum tuberosum L.), a $28{ }^{\circ} \mathrm{C}$ associadas à umidade relativa de $87 \pm$ 4\%. Segundo Sherf \& Macnab (1986), a umidade favorece a esporulação de $A$. solani que aumenta à medida que a temperatura aumenta para $27^{\circ} \mathrm{C}$ durante o período de molhamento foliar.

Além das condições climáticas, a fase maturação fisiológica do tomateiro (Barratt \& Richards, 1944) e o regime de controle podem, também, afetar o desenvolvimento da pintapreta. O tratamento semanal (SCV) proporcionou progresso mais lento da doença que os tratamentos que seguiram regimes de pulverização impostos pelos sistemas de previsão. Todos os tratamentos, independentemente do regime de pulverização, mantiveram a severidade abaixo dos 15\% de área foliar lesionada até os 50 dias após o transplantio (DAT) (Figura 2). Entre 28 e 35 DAT foram observadas taxas de infecção negativas (Figura 1A). Esta tendência pode ser atribuída ao fato de que este período correspondeu com a fase durante a qual a taxa de crescimento vegetativo da planta foi maior do que a taxa de aumento da intensidade da doença. Com o acréscimo de novos tecidos sadios, a proporção de tecido doente pode decrescer, apesar de poder haver um aumento da quantidade total de doença na planta (Campbell \& Madden, 1990; Bergamin Filho, 1995). O aumento na taxa de progresso da doença, observado entre 50 e 77 DAT - período que coincidiu com a época de formação de um número maior de cachos de frutos -, pode ser atribuído ao aumento na susceptibilidade do hospedeiro. Horsfall \& Heuberger (1942), citados por Rotem (1994), demonstraram que a susceptibilidade do tomateiro a A. solani é condicionada pela razão fruto por folha. Segundo estes autores, o aumento da carga de frutos resulta no aumento da susceptibilidade da planta e, por conseqüência, no aumento
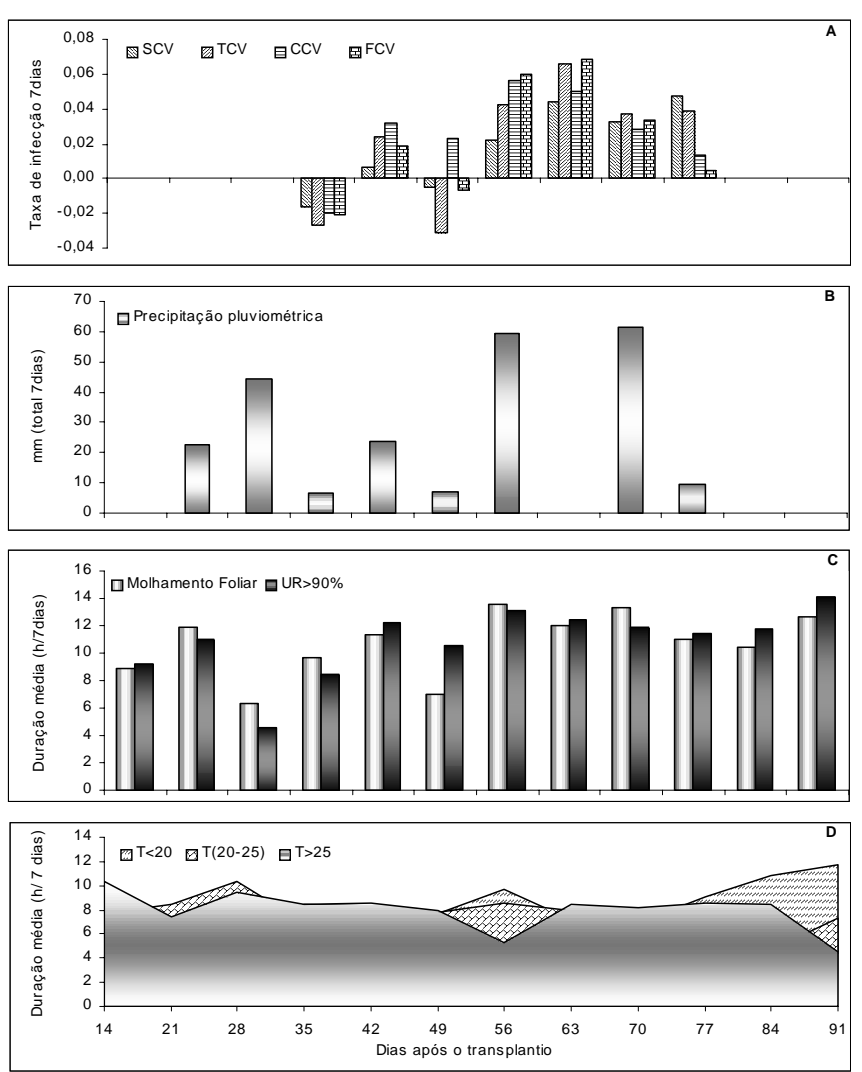

FIG. 1 - Taxa de infecção aparente semanal da pinta-preta do tomateiro (Lycopersicon esculentum) referente aos diferentes tratamentos (A); precipitação pluviométrica (B); molhamento foliar e umidade relativa $>90 \%$ (C); e temperatura em ${ }^{\circ} \mathrm{C}(\mathrm{D})$. 


\section{P.A. Paul et al.}

da intensidade da doença.

No tocante aos modelos de crescimento, o modelo logístico foi aquele que melhor se ajustou aos dados de severidade. Para todos os tratamentos, este modelo apresentou os maiores valores de $\mathrm{R}^{2}$ e os menores valores de QMR* quando comparado com o de Gompertz e o monomolecular (Tabela 3). Além disso, entre os três modelos, os valores dos resíduos do modelo logístico, plotados em função dos valores previstos, apresentaram a distribuição menos tendenciosa. Com base nisso, o modelo logístico foi escolhido e os dados de severidade foram utilizados para obter as taxas $\mathbf{r}$ por meio de analise de regressão não-linear, e estas taxas, juntamente com a AACPD, foram utilizadas para comparar as epidemias (Tabela 4).

Neste ensaio, observou-se que o SCV, no qual a primeira aplicação foi realizada aos 14 DAT e um total de dez aplicações foi feito ao longo do ciclo da cultura, proporcionou menor taxa de progresso da doença e AACPD que os demais tratamento (Tabela 4). Entre os tratamentos baseados nos modelos de previsão, os tratamentos 2 (TCV) e 4 (FCV), nos quais foram realizadas seis e quatro aplicações seguindo os regime impostos pelos sistemas de previsão TOMCAST (VSD 20) e FAST, respectivamente, se destacaram, proporcionando valores de taxa e AACPD que não diferiram estatisticamente do tratamento semanal.

Os resultados obtidos neste trabalho mostram que as aplicações de fungicidas com base nos sistemas de previsão TOMCAST e FAST podem proporcionar controle semelhante
TABELA 3 - Resumo da análise de regressão linear ${ }^{1}$ usada para avaliar o ajustamento dos três modelos de crescimento aos dados de severidade da pinta-preta do tomateiro (Lycopersicon esculentum), referente aos diferentes tratamentos

\begin{tabular}{|c|c|c|c|c|c|c|}
\hline \multirow[b]{2}{*}{ TRAT } & \multicolumn{2}{|c|}{ Gompertz } & \multicolumn{2}{|c|}{ Logístico } & \multicolumn{2}{|c|}{ Monomolecular } \\
\hline & $\begin{array}{l}\mathbf{R}^{2(*)} \\
(\%)\end{array}$ & QMR & $\begin{array}{l}\mathbf{R}^{2(*)} \\
(\%)\end{array}$ & QMR & $\begin{array}{l}\mathbf{R}^{2(*)} \\
(\%)\end{array}$ & QMR \\
\hline SCV & 59,68 & 0,00092 & 61,99 & 0,00079 & 54,64 & 0,00126 \\
\hline TCV & 75,38 & 0,00096 & 78,53 & 0,00077 & 68,21 & 0,00147 \\
\hline $\mathrm{CCV}$ & 92,90 & 0,00057 & 93,79 & 0,00050 & 88,62 & 0,00099 \\
\hline $\mathrm{FCV}$ & 82,21 & 0,00099 & 83,92 & 0,00086 & 76,71 & 0,00146 \\
\hline
\end{tabular}

${ }^{(*)} \mathrm{R}^{2}=$ coeficiente de determinação para ajuste entre valores observados e previstos de severidade, QMR = quadrado médio do resíduo.

TABELA 4 - Número de pulverizações, época da primeira pulverização, Área abaixo da curva de progresso da doença (AACPD) e taxa de infecção aparente da pinta-preta do tomateiro (Lycopersicon esculentum) (r)

\begin{tabular}{|c|c|c|c|c|}
\hline \multirow{2}{*}{ TRAT } & \multicolumn{2}{|c|}{ PULVERIZACC̃̃O } & \multirow{2}{*}{ AACPD $* *$} & \multirow{2}{*}{$\mathbf{r} * *$} \\
\hline & № & 1' $($ DAT)* & & \\
\hline SCV & 10 & 14 & $839,95 \quad \mathrm{~B}$ & $0,0233 \quad \mathrm{~B}$ \\
\hline TCV & 6 & 20 & $894,61 \mathrm{AB}$ & $0,0294 \mathrm{AB}$ \\
\hline $\mathrm{CCV}$ & 3 & 57 & $1081,43 \mathrm{~A}$ & $0,0333 \mathrm{~A}$ \\
\hline $\mathrm{FCV}$ & 4 & 35 & $942,65 \mathrm{AB}$ & $0,0323 \mathrm{AB}$ \\
\hline C.V. $\%$ & & & 10,17 & 15,37 \\
\hline
\end{tabular}

*/Dias após o transplantio;

**/Nas colunas, as médias seguidas pela mesma letra não diferem entre si ao nível de $5 \%$ de probabilidade pelo teste de Tukey

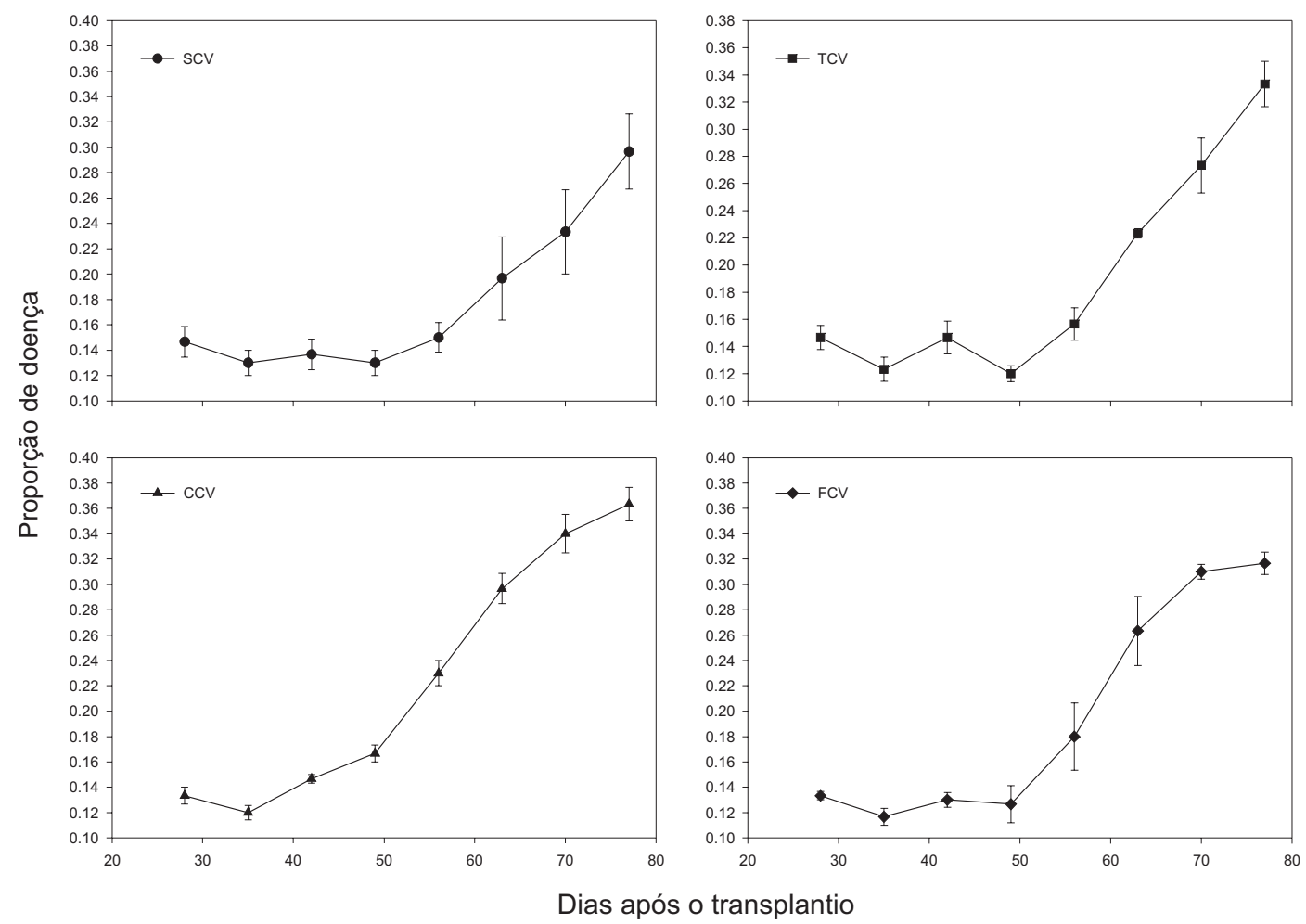

FIG. 2 - Curvas de progresso da pinta-preta do tomateiro (Lycopersicon esculentum) para os diferentes tratamentos. Tratamentos: 1 - Semanal (SCV); 2 - TOMCAST (TCV); 3 - CUFAST (CCV); 4 - FAST (FCV). 
Epidemiologia comparativa da pinta-preta do tomateiro sob quatro...

à aplicação semanal, pois o número e freqüência de aplicação de fungicidas nem sempre são os fatores mais importantes para o controle eficiente de uma doença. A aplicação de fungicidas com base nas condições climáticas, seguindo os regimes de pulverização impostos pelo acúmulo de VSD 20 do modelo TOMCAST (Bolkan \& Reinert, 1994; Gleason et al., 1995; Paul, 1999) e pelo modelo FAST (Madden et al., 1978; Pennypacker et al., 1983; Macnab et al., 1983), pode resultar na redução do número de aplicação sem comprometer o controle da pinta-preta do tomateiro.

\section{REFERÊNCIAS BIBLIOGRÁFICAS}

BARRATT, R.W. \& RICHARDS, M.C. Physiological maturity in relation to alternaria blight in the tomato. Phytopathology 34:997. 1944 (Abstract).

BERGAMIN FILHO, A. Conceitos e objetivos. In: Bergamin Filho, A., Kimati, H. \& Amorim, L. (Eds.) Manual de Fitopatologia: Princípios e Conceitos. São Paulo. Agronômica Ceres. 1995. pp.540553.

BOFF, P. Epidemiologia e controle químico da mancha-de-estenfílio (Sthemphylium solani Weber) e da pinta-preta (Alternaria solani Jones \& Grout), em dois sistemas de condução do tomateiro (Lycopersicon esculentum Mill). (Tese de Mestrado). Viçosa. Universidade Federal de Viçosa. 1988.

BOLKAN, H. A. \& REINERT, W.R. Developing and implementing IPM strategies to assist farmers: an industry approach. Plant Disease 78:545-550. 1994.

CAMPBELL, C.L. \& MADDEN, L.V. Introduction to Plant Disease Epidemiology. New York: John Wiley \& Sons Inc. 1990.

GLEASON, M.L., MACNAB, A.A., PITBLADO, R.E., RICKER, M.D. EAST, D.A. \& LATIN, R.X. Disease-warning systems for processing tomatoes in Eastern North America: are we there yet? Plant Disease 79:113-121. 1995.

JAMES, W.C., SHIH, C.S., CALLBECK, L.C. \& HODGSON, W.A. Interplot interference in field experiments with late blight of potato (Phytophthora infestans). Phytopathology 63:1269-1275. 1973.

JONES, J.P. Early blight. In: Jones, J.B., Jones, J.P., Stall, R.E. \& Zitter, T.A. (Eds.) Compendium of Tomato Diseases. St. Paul. APS Press. 1991. pp.13-14.

KRANZ, J. Enfermedades de los cultivos tropicales. In: Krans, J., Schmutterer, H. \& Koch, W. (Eds.) Enfermedades, Plagas e Malezas de los Cultivos Tropicales. Hamburg. Verlag Paul Parey. 1982. pp.1246.

MACNAB, A.A., PENNYPACKER, S.P. \& JURCHAK, T. Comparison of FAST with a regular schedule for tomato early blight control, 1982. Fungicide and Nematicide Tests 38:110-111. 1983.

MADDEN, L.V., PENNYPACKER, S.P. \& MACNAB, A.A. FAST a forecast system for Alternaria solani on tomato. Phytopathology 68:1354-1358. 1978.

MAFFIA, L.A., MARTINS, M.C. del P. \& MATSUOKA, K. Doenças do tomateiro. Informe Agropecuário 6:42-60. 1980.

NUNES, M.A.L., ZAMBOLIM, L., CHAVES, G.M. \& MIZUBUTI, A. Efeito da temperatura e densidade de inóculo de Alternaria solani sobre a taxa de progresso da pinta-preta em diferentes cultivares de batateira. Fitopatologia Brasileira 9:327. 1984 (Resumo).

PAUL, P.A. Sistemas de manejo da pinta-preta (Alternaria solani (Ellis \& Martin) Jones \& Grout) do tomateiro. (Tese de Mestrado). Viçosa. Universidade Federal de Viçosa. 1999.

PENNYPACKER, S.P., MADDEN, L.V. \& MACNAB, A.A. Validation of an early blight forecasting system for tomatoes. Plant Disease 67:287-289. 1983.

PITBLADO, R.E. The development and implementation of TOMCAST - a weather-timed fungicide spray program for field tomatoes. Ministry of Agriculture and Food, Ridgetown College of Agricultural Technology, Ridgetown. 1992.

ROTEM, J. The Genus Alternaria. Biology, Epidemiology, and Pathogenicity. St. Paul. APS Press. 1994.

SANDLAN, K.P. \& ZITTER, T.A. Scheduling fungicide sprays to control tomato early blight using a modified FAST forecasting program. Plant Pathology Extension Report 1989/1993, Cornell University. New York. 1993.

SHERF, A.F. \& MACNAB, A.A. Tomato. In: Sherf, A.F. \& Macnab, A.A. (Eds.) Vegetable Diseases and Their Control. New York. John Wiley \& Sons. 1986. pp. 599-696.

VALE, F.X.R., ZAMBOLIM, L., CHAVES, G.M. \& CORREIA, L.G. Avaliação fitossanitária da cultura do tomateiro em regiões produtoras de Minas Gerais e Espírito Santo. Fitopatologia Brasileira 17:211. 1992 (Resumo).

Van der PLANK, J.E. Plant Diseases: Epidemics and Control. New York. Academic Press. 1963.

ZADOKS, J.C. \& SCHEIN, R.D. Epidemiology and Plant Disease Management. New York. Oxford University Press. 1979. 